\title{
ZONIFICACIÓN PAISAJÍSTICA DE LA HACIENDA EL OCASO, QUIMBAYA, QUINDÍO
}

\author{
ZONING LANDSCAPE OF THE HACIENDA EL OCASO, QUIMBAYA, QUINDÍO
}

\author{
Nathalia Aguilar-Isaza ${ }^{1}$, Diana Carolina López-Obando ${ }^{1}$, Alvaro Botero-Botero ${ }^{2}$, Germán Darío Gómez Marín ${ }^{3}$
}

${ }^{1}$ Programa de Biología, Universidad del Quindío. ${ }^{2}$ Fundación Neotrópica-Colombia. ${ }^{3}$ Reserva El Ocaso. CIBUQ. Universidad del Quindío

Fecha de recibido: Febrero 3 de 2010

Fecha de aceptado: Junio 9 de 2010

Correspondencia: Centro de Estudios e Investigaciones en Biodiversidad y Biotecnología (CIBUQ). Bloque de Ciencias Básicas.

Universidad del Quindío. Armenia, Quindío. Correo postal 630004. Correo electrónico: germandario@uniquindio.edu.co

\section{RESUMEN}

Dentro de la Hacienda El Ocaso, encontramos la Reserva natural la montaña del Ocaso que actualmente es el mayor relicto en conservación presente en la zona baja del departamento del Quindío con 148,84 hectáreas, con el fin de contribuir al modelo de conservación que actualmente adelanta la Universidad del Quindío en dicha área, se planteó como objetivo realizar la zonificación paisajística y caracterización del uso del suelo dentro de la reserva. Desde marzo de 2008 hasta febrero de 2010, se actualizó la cartografía del área, tomando como base una fotografía aérea y con actualización con visitas a campo. Posteriormente, se elaboraron mapas de cobertura vegetal y uso del suelo actual de la Hacienda El Ocaso, mapas de zonas de interés en investigación y conservación, proponiéndose una serie de corredores boscosos que comunican áreas fragmentadas con el fragmento mayor. Dentro de las coberturas vegetales naturales, se evidenciaron tres tipos: guadual, conformado principalmente por Guadua angustifolia, bosque secundario sin la presencia de G. angustifolia y bosque mixto donde se presenta una combinación de guadua y bosque secundario. Actualmente se evidencia un aumento en los últimos 10 años más del $50 \%$ del guadual tanto dentro como fuera de reserva. El bosque protegido ha aumentado en 42,84 ha desde la última medida realizada en el año 2007.

Palabras clave: cartografía de vegetación, Zonificación paisajística, reservas naturales, uso del suelo, diversidad vegetal, andes de Colombia

\section{ABSTRACT}

The natural reserve "The Mountain of Ocaso" is part of the Hacienda El Ocaso. Actually, it is the mayor relict of the natural forest preserved in the low land of the department of Quindio with $148.84 \mathrm{ha}$. In order to contribute to the model of conservation that the University of Quindio is realizing in this area, as an objective to be realized, it is proposed to realize characterization and landscaping zonification of the land use within the reserve. From March of 2008 until February of 2010 the cartography of the area was actualized, taking as a base one aerial photo and the exploration of the site of the interest. The maps of the vegetation cover and the actual land use were elaborated as well as maps of the areas of the interest for investigation and conservation, and a series of forest corridors which communicate fragmented areas with the biggest fragment of forest. Within the natural vegetation the presence of Guadua angustifolia and secondary vegetation without the presence of G. angustifolia is found. In some places of the secondary forest it is observed the combination of the presence of the $G$. angustifolia and the natural vegetation. In the last ten years it is observed an increment of more than $50 \%$ of the Guadua angustifolia within the reserve and also outside of the reserve. The protected area of the forest increased up to 42.84 ha from the time of the last record in 2007.

Key Words: Landscaping zonification, natural reserve, land use, vegetable diversity, Vegetation Cartography, Andes of Colombia 


\section{INTRODUCCIÓN}

En Colombia, las coberturas boscosas tienden a disminuir con el transcurso del tiempo. En el periodo comprendido entre 1986 a 1991 se perdieron 622,36 ha a una tasa promedio anual de 0,14\%. Esta pérdida se incrementó para el periodo 1994-2001 en un promedio de 0,18\%; mientras que los agroecosistemas entre 1986-2001 incrementaron a 0,17\% anual. La zona donde los agroecosistemas presentan mayor porcentaje de crecimiento es la Andina, la cual para el 2001 llegó a representar el 13,33\% del total del territorio nacional (1), siendo esta región uno de los ecosistemas más afectados puesto que en la actualidad solo son preservados menos del $30 \%$ de su hábitat original $(2,3,4,5)$.

Dentro de la región andina, la reserva natural La Montaña del Ocaso no ha sido ajena a la perdida de cobertura vegetal. La reserva se encuentra aislada desde principios del siglo pasado, en 1954 presentaba un área de 262,9 ha pero en 50 años perdió el $42 \%$ de la cobertura boscosa. Además en los años siguientes, el bosque fue altamente intervenido y casi todos los árboles maderables fueron extraídos hasta hace aproximadamente 10 años, cuando inició el comodato con la Universidad del Quindío, quien lo ha protegido (6). Actualmente, La Reserva El Ocaso es el mayor relicto bajo una figura jurídica de conservación presente en la zona baja del departamento del Quindío y como tal posee una alta representatividad de especies vegetales (7) y es una muestra viviente de los ecosistemas naturales de esta zona.

El objetivo de este trabajo fue la caracterización de la cobertura y usos del suelo en la Hacienda El Ocaso, generando lineamientos para el manejo, conservación y aprovechamiento del paisaje.

\section{Área de estudio}

\section{MÉTODOS}

La Hacienda El Ocaso, está ubicada en la vereda el Laurel, municipio de Quimbaya, departamento del Quindío, a 40 34`08” N y 75으 51 03” O a 970 msnm. Dentro de la hacienda se encuentra la reserva natural "La Montaña del Ocaso", la cual la familia Vallejo entrega en comodato a la Universidad del Quindío, el 25 de Agosto de 2000, con una extensión aproximada actual de 148,84 ha. Esta reserva hace parte de la zona baja de la microcuenca del río Roble, localizado en la confluencia del río La Vieja. El relieve predominante es plano a ondulado, con pendientes que varían desde el $5 \%$ al $25 \%$. La vegetación nativa de la zona es una transición entre el bosque húmedo premontano (bhPM) y bosque seco Tropical (bs-T), con algunos elementos de bosque muy húmedo premontano (bmh-PM) (8). La temperatura promedio es de $24^{\circ} \mathrm{C}$. La Hacienda El Ocaso limita al norte con las haciendas La Cascada, El Dobaide y La Floresta, al sur con el río Roble, al este con la hacienda La Española, y al oeste con el río La Vieja (9).

\section{Fase de campo}

Para la realización de la cartografía de cobertura vegetal, se utilizó como base de trabajo una fotografía aérea a blanco y negro ortorectificada del año 2003 en la cual se trazaron los linderos, con ayuda de fotografías aéreas tomadas por un ultraliviano en el año 1999 y el programa Google Earth (c) fue elaborado un mapa inicial de interpretación visual de estructuras parecidas por su tono, color, textura, forma y tamaño $(10,11)$. La identidad de todas las estructuras vegetales fue corroborada con visitas a campo, con lo que se actualizó dicho mapa con la toma de diferentes coordenadas con un GPS Garmin Etrex ${ }^{\circledR}$ y se trabajo a una escala de 1:8000. El manejo de la cartografía se realizó con los programas MapSource y ARCVIEW GIS $3.2{ }^{\circledR}$ (Environmental Systems Research Institute, Redlands, California).

Para clasificar los tipos de coberturas vegetales se utilizaron tres criterios: primero la presencia dominante de la especie $G$. angustifolia se le denominó cobertura de guadual, al bosque sin presencia de $G$. angustifolia se le denominó bosque secundario, y a la cobertura que presenta mezcla de ambas formaciones vegetales, se llamó bosque mixto. En la caracterización del uso del suelo se utilizaron las siguientes categorías: zonas sembradas con pasto para ganado vacuno, viviendas, vías principales y vías secundarias. Para trazar el curso de los cuerpos de agua y los senderos dentro de la reserva se utilizó como base cartografía previamente diseñada en años anteriores (12).

\section{RESULTADOS Y DISCUSIÓN}

\section{Uso del suelo, cobertura e hidrografía}

La hacienda El Ocaso presenta un área total de 790,87 ha. de las cuales 457,58 ha. (57,86\%), están destinadas a la producción ganadera, 66,14 ha. $(8,36 \%)$ en 
guaduales para aprovechamiento forestal y 148,84 ha. $(18,82 \%)$ bajo la figura de conservación estricta dentro de La Reserva Natural La Montaña del Ocaso (Figura 1 y Tabla 1); se tiene proyectado la reforestación de 13,93 ha de potreros (Figura 2 y Tabla 1 ). El área de guaduales ha aumentado en los últimos 10 años en más del $50 \%$, tanto dentro de la hacienda como en el área de conservación, dado que para el año de 1989 el área de guadua fuera de la reserva era de 59,21 ha y dentro de la reserva de 17,47 ha. (8).

El bosque protegido en la hacienda El Ocaso actualmente cuenta con un área de 148,84 ha, generando un aumento del área boscosa del 42,84 ha desde la última medida basada en fotografías aéreas realizada por otros autores en el año 2007, donde el área era de 106 ha. (6). Actualmente, la cobertura

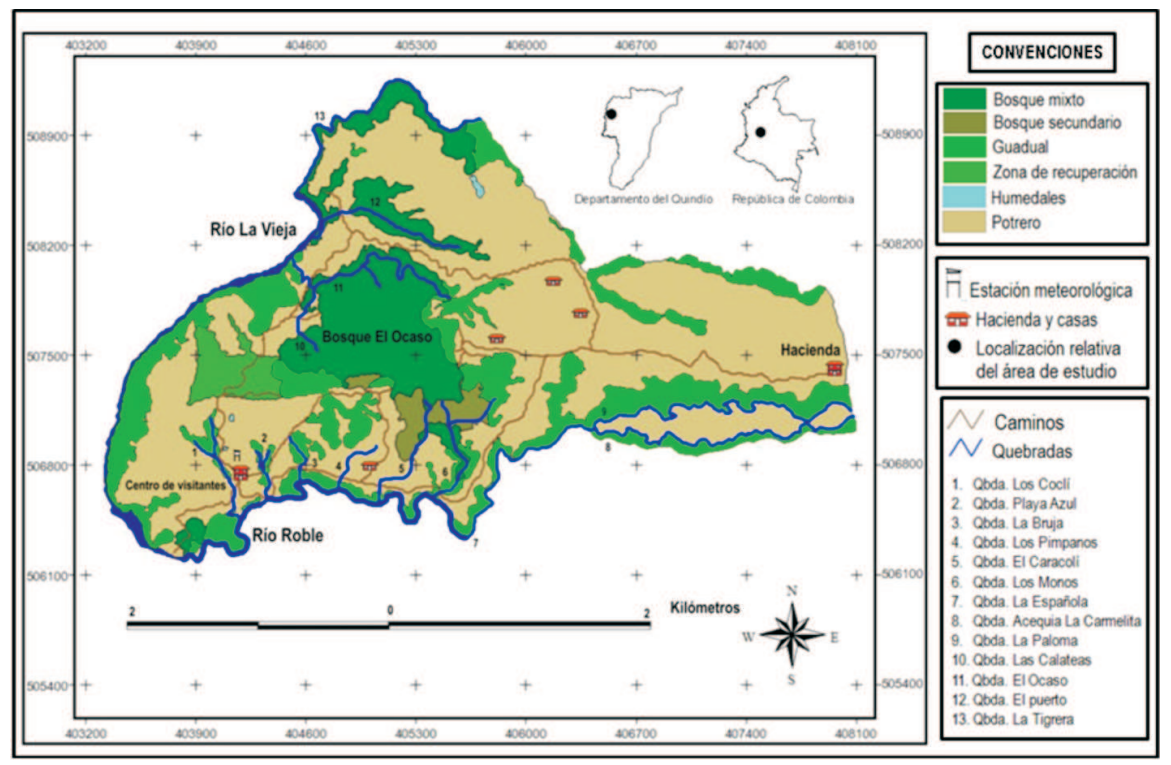

Figura 1. Mapa de la Reserva Natural la montaña del Ocaso con su respectiva cobertura e hidrografía, Quimbaya, Quindío.

Tabla 1. Tipos de usos del suelo en la Hacienda El Ocaso.

\begin{tabular}{lrr}
\hline \multicolumn{1}{c}{ T ipos de usos } & H ectár eas (ha) & Por centaje (\% ) \\
\hline G anadero & 457,58 & 57,86 \\
Guadual para aprovechami ento & 66,14 & 8,36 \\
Guadual & 64,42 & 8,15 \\
B osque mix to & 37,08 & 4,69 \\
Conservación estricta & 148,84 & 18,82 \\
H umedales & 0,75 & 0,09 \\
B anco de germoplasma & 0,39 & 0,05 \\
Corredores & 1,73 & 0,22 \\
Á reas para reforestar & 13,93 & 1,76 \\
T OT A L & $\mathbf{7 9 0 , 8 7}$ & $\mathbf{1 0 0 \%}$ \\
\hline
\end{tabular}


dominante es el bosque mixto con el $51,66 \%(76,90$ ha.), seguida por los guaduales con $25,13 \%$ ( 37,41 ha.), la zona de recuperación con $12,74 \%(18,96$ ha.) y por último el bosque secundario con $10,46 \%(15,57$ ha.) (Figura 1).

El incremento en todas las coberturas vegetales que se ha dado en los últimos años en la hacienda el Ocaso, es debido al modelo de conservación adoptado por la Universidad del Quindío basado en el proceso investigación y extensión a la comunidad del centro de investigaciones en biodiversidad de la Universidad del Quindío CIBUQ (13). Además, la condición de conservación de los guaduales en la hacienda El Ocaso, ha favorecido los procesos de regeneración e incorporación de la flora asociada a este tipo de ecosistemas, dando como origen la conformación de zonas conocidas como bosque mixto, donde a pesar de la dominancia que posee $G$. angustifolia, aparece toda una gran diversidad de especies asociadas (7).

La cobertura de la hacienda El Ocaso en especial aquellas zonas conformadas por guaduales y que se encuentran aledañas a la casa principal, tiene el potencial para dar inicio a un plan de aprovechamiento sostenible de guaduales, acogiendo los criterios técnicos definidos por la autoridad ambiental (CRQ). Por otro lado, otras áreas de guaduales, deben permanecer sin ningún tipo de intervención antrópica y con el único objetivo de conservar muestras de bosques de guadua sin intervención, que generen condiciones favorables para el desarrollo de un gran número de especies asociadas a la G. angustifolia.

Se recomienda que en áreas cercanas al cauce del río La Vieja (a partir de las coordenadas 18N 404600/ 508900 ) y del río Roble (a partir de las coordenadas $18 \mathrm{~N} 408100 / 507500)$ donde se presentan pendientes mayores a $25^{\circ}$ se implemente modelos alternativos de aprovechamiento ganadero tales como: ganadería sostenible y agroecosistemas silvopastoriles que minimicen el impacto sobre el suelo de esta actividad y no entren en conflicto con los intereses económicos de los propietarios de la Hacienda.

La Reserva la Montaña del Ocaso posee un potencial hídrico representado en 13 quebradas que nacen o recorren parte de su territorio (Figura 1). Nueve de ellas aportan aguas al río Roble en la parte baja de su cuenca, mientras cuatro de ellas (La Tigrera, El Puerto, El Ocaso y Las Calateas) lo hacen sobre el río La Vieja, que corresponde a la mayor cuenca hidrográfica del departamento del Quindío. Estas 13 quebradas sumadas a los ríos Roble y La Vieja, generan un microclima en la zona, que favorece la presencia de algunas especies vegetales como el grupo de las Bromelias y epifitas, propias de zonas con tasas de humedad alta.

\section{Aportes al proceso de conservación de la Reserva Natural la Montaña del Ocaso}

Para evitar el efecto de insularización y disminuir al máximo la distancia entre fragmentos, se ha propuesto la conformación de seis corredores que garantizan la conectividad entre los diferentes fragmentos que hacen parte del área de reserva para facilitar la movilidad de mamíferos terrestres y arbóreos, aves y herpetos. Además, se establecen algunas zonas con potencial para la reforestación con el fin único de aumentar cobertura protegida (Figura 2).

Como estrategia para promover la conservación de especies forestales nativas de la zona cálida del departamento del Quindío, La Reserva Natural la Montaña del Ocaso, ha establecido en un área aproximada de 0,39 ha, un banco de germoplasma in situ (Figura 3) de alrededor de 32 especies forestales, con el objeto de mantener el germoplasma de estas especies y hacer ensayos sobre su propagación. Actualmente este banco, apoya iniciativas de manejo del paisaje como el establecimiento de corredores biológicos y la restauración de áreas degradadas. Con esto la Reserva El Ocaso ha procurado mantener un representatividad de las especies forestales propias de la zona cálida del Quindío.

Otra estrategia que se ha planteado para el de manejo del paisaje en la Reserva, es implementar procesos de restauración (Figura 2) con especies nativas e incluso favorecer la regeneración natural, destinando para ello 13,93 ha. Esta iniciativa busca aportar a la conectividad entre fragmentos, la protección de zonas con potencial hídrico (humedales y quebradas) y a la estabilización de áreas con riesgo de erosión.

En principio se han definido como especies con potencial para reforestación las siguientes: 


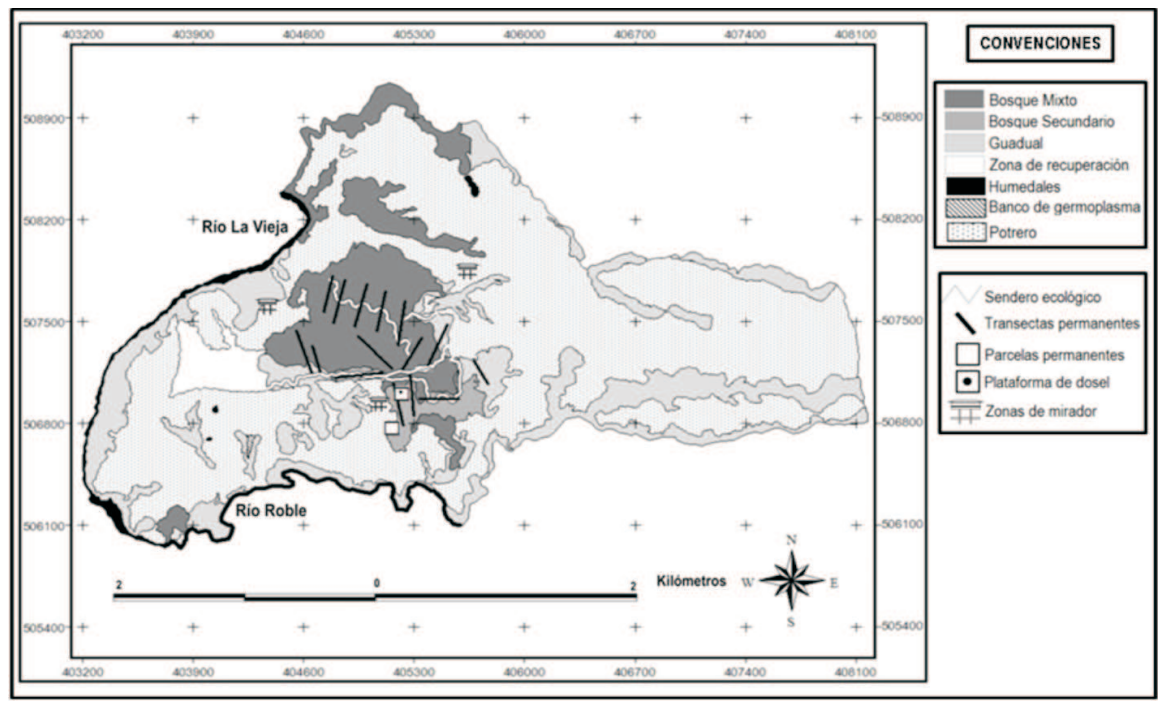

Figura 2. Mapa de potencial de conservación de la Reserva Natural la montaña del Ocaso, Quimbaya, Quindío

Anacardium excelsum (Caracolí), Pseudolmedia rigida (Míspero de Monte), Celtis iguanae (Uña de gato), Luhea seemanii (Guasimo negro) y varias especies del género Ficus. El principal criterio para la selección de estas especies, está basado fundamentalmente en el aporte y la oferta representada en la producción de hojas, flores y frutos, para la fauna silvestre.

Con el fin de general procesos productivos en torno a las áreas boscosas de la Hacienda El Ocaso, se ha propuesto una delimitación de proximadamente 66,14 ha de guadual (Figura 2), para que sean incorporadas dentro de un plan de aprovechamiento forestal sostenible, que pueden enmarcarse dentro de uso doméstico o comercial.
Considerando la importancia que tiene los rodales de G. angustifolia, como soporte estructural para la fauna que posee la reserva, es necesario que aquellos rodales que se definen con una vocación para su aprovechamiento, tengan una intervención de bajo impacto representada en porcentajes mínimos (20$25 \%)$ de extracción y periodos prolongados de descanso entre un corte y otro (mínimo cada 2 años).

Teniendo en cuenta la importancia hídrica que posee la Hacienda El Ocaso, representada en 13 quebradas y el límite con los ríos de La Vieja y El Roble, en el área total de ésta se han definido 0,75 ha, que por su topografía, tipo de suelo y nivel freático, tienen potencial para ser destinadas como zonas de humedales (Figura 1 y 2). Estos humedales, favorecerían la presencia de fauna y flora asociada a este tipo de condiciones. Adicionalmente, se garantizaría reservorios naturales

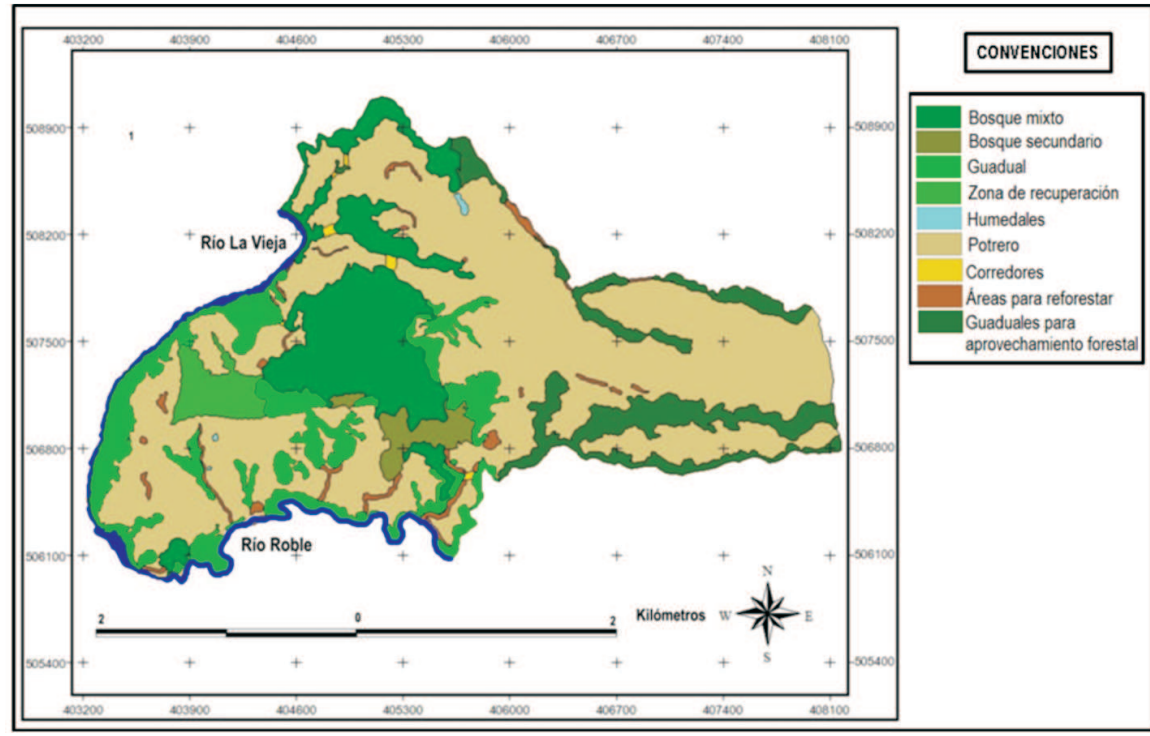

Figura 3. Mapa de investigación de la Reserva Natural la montaña del Ocaso, Quimbaya, Quindío. 
de agua para las actividades productivas de la Hacienda.

Por otro lado, para el momento en que se decida incorporar el uso ecoturístico de la Hacienda, se tienen planteadas tres zonas, en las cuales se pueden ubicar miradores, que de acuerdo a la topografía permiten tener una visión panorámica del paisaje de la reserva EI Ocaso y de gran parte de la microcuenca del río de La Vieja (Figura 3). Además, se cuenta con una infraestructura básica, para ofrecer alojamiento para visitantes (Figura 1) y un sendero de interpretación ambiental (Figura 3). Sin embargo, para poder desarrollar un modelo de ecoturismo en la reserva, es indispensable la realización de un plan de manejo para minimizar los impactos que esta actividad puede generar sobre este tipo de ecosistema.

\section{Monitoreo e Investigación}

La Reserva La montaña del Ocaso cuenta actualmente con la siguiente estructura física para apoyar procesos de investigación y monitoreo: un Centro de visitantes (Figura 1) (Playa Azul), ubicado en el costado sur occidente de la reserva, en el cual estudiantes, docentes e investigadores se alojan por periodos de tiempo necesarios; una estación meteorológica (Figura 1) que registra datos climatológicos como: precipitación, temperatura media, humedad Relativa e intensidad lumínica, teniendo un registro más o menos continuo desde el año 1986.

De igual modo dentro del componente de monitoreo e investigación en la Reserva El Ocaso se dispone actualmente dos parcelas permanentes (una en zona de interior y otra en zona de borde), cada una de $100 \mathrm{x}$ $25 \mathrm{~m}(0,25 \mathrm{ha})$, como estrategia para evaluar la dinámica de la composición, fenología y estructura florística de esta área. Dentro de una de estas parcelas se halla una plataforma de dosel, ubicada a una altura de 23 m en la especie arbórea Pseudolmedia rigida, que tiene una altura total de $26 \mathrm{~m}$. Así mismo, se han ubicado 15 transectas lineales permanentes por todo el bosque con una distribución aleatoria, las cuales poseen longitudes que oscilan entre 175 a 300 m (14), en éstas se pueden diseñar una gran cantidad de investigaciones. (Figura 3)

Actualmente, la reserva natural posee las siguientes locaciones que se utilizan en procesos de educación ambiental con jóvenes y adultos: un sendero de interpretación y un centro de interpretación ambiental. El sendero tiene una extensión de $2 \mathrm{~km}$, el cual atraviesa el área del bosque del extremo nororiente al sur-occidente (figura 2). Por consiguiente, estas actividades se realizan sin necesidad de ocupar o intervenir en otros lugares que por su topografía e importancia ecosistémica, deben tener un uso restringido o limitado únicamente a procesos de investigación.

\section{AGRADECIMIENTOS}

Este trabajo fue llevado a cabo dentro del proyecto de grado de las dos primeras autoras, en el programa de Biología de la Universidad del Quindío. Queremos agradecer a las siguientes instituciones y personas: Centro de Investigaciones en Biodiversidad de la Universidad del Quindío (CIBUQ), Reserva Natural La Montaña del Ocaso, Fundación Neotrópica-Colombia por la colaboración con los equipos para las labores de campo. A Carolina Gómez Posada quien facilito la fotográfica aérea. A los guardabosques de la Reserva Natural, Daniel Rodríguez y a Jamith Claros por el acompañamiento en campo. A Luis Alfonso Aguilar Trujillo, Juan Diego Martínez, Jessica López Obando, Guillermo Alonso Cárdenas y a todos los que colaboraron activamente en los muestreos.

\section{BIBLIOGRAFÍA}

1 IDEAM (eds) (2004). Informe anual sobre el estado del medio ambiente y los recursos naturales renovables en Colombia. Imprenta Nacional de Colombia. 256 pp.

2. Etter, A. (1993). "Diversidad ecosistémica en Colombia hoy"., En: CEREC - Fundación Alejandro Ángel (FAA), 1993. Nuestra diversidad biológica (47-6). Bogota: CEREC-FAA.

3. Kattan G. H. \& Alvarez-López H. (1996). "Preservation and management of biodiversity in fragmented landscapes in the Colombian Andes"., En: Schelhas J. \& R. Greenber (Eds.) Forest Patches in Tropical Landscapes (3-18). Washington: Island Press.

4. Etter, A. \& Van Wyngaarden. (2000). "Patterns of Landscape Transformation in Colombia, with Emphasis in the Andean Region". Ambio. (29), 432-439.

5. Cavelier J., Lizcano D. \& Pulido M. T.. 2001. “Colombia”., En: Kappelle, M. \& Brown, A. (Eds.). Bosques Neotropicales de Montaña.(443-496). San Jose, Costa Rica: INBio, World Conservation Monitoring Center, World Conservation Union. 
6. Gómez-Posada, C. \& J. M. Londoño. (2007). Evaluación del estado de la población del mono aullador rojo (Alouatta seniculus) en la reserva La Montaña Del Ocaso. Informe presentado al CIBUQ, Universidad Del Quindío. Fundación EcoAndina / WCS Colombia, Cali.

7. Gómez G. (2007). Composición florística y desfonde en zonas de interior y borde de dos fragmentos de selva subandina del departamento del Quindío. Trabajo de Investigación para optar al título de Magister en Biología Vegetal. Universidad del Quindío. Universidad Tecnológica de Pereira. Universidad de Caldas. Maestría en Biología Vegetal. Armenia.

8. Holdridge L. (1978). Ecología basada en zonas de vida. Quinta reimpresión. San José, C.R. Instituto interamericano de cooperación para la agricultura. $216 \mathrm{p}$.

9. Villa R., \& Vila J. (1998). "Recopilación y revisión cartográfica de la selva "La Montaña del Ocaso”, Quimbaya, departamento del Quindío". Revista de investigaciones Universidad del Quindío. 4 (13), 29-36.

10. Ojasti, J. (2000). Manejo de fauna Silvestre Neotropical. F. Dallmeier (Ed.) Washington, D.C: SIMAB series No. 5 Smitsonian institution/MAB program.

11. Newton, A. (2007). Forest ecology and conservation. A handbook of techniques. New York: Oxford University Press Inc.

12. Agudelo, C. y Gómez-Marín, G. (2001). Reserva Natural La Montaña del Ocaso: un nuevo modelo de conservación. Monografías de la Flora Andina. Armenia: Universidad del Quindío.

13. Agudelo C., \& Vélez C. (2001). Reserva natural la montaña del Ocaso: un nuevo modelo de conservación. Monografías de la flora andina Vol. 3. Herbario HUQ, Universidad del Quindío.

14. Aguilar-Isaza, N. y López-Obando D. (2009). Estado poblacional actual y hábitat del perezoso de dos dedos (Choloepus hoffmanni, Peters, 1858) en la Reserva Natural la Montaña del Ocaso, Quimbaya (Quindío), Colombia. Trabajo de grado presentado como requisito para optar al título de Biólogo. Facultad de Ciencias Básicas y Tecnológicas, Programa de Biología. Universidad del Quindío. Quindío. 